\title{
Influence of plasma boundary shape on helical core/long-lived mode in tokamak plasmas
}

$\operatorname{AUTHOR}(S)$ :

Nakamura, Y.; Ishizawa, A.; Ishida, Y.

\section{CITATION:}

Nakamura, Y....[et al]. Influence of plasma boundary shape on helical core/long-lived mode in tokamak plasmas. Physics of Plasmas 2020, 27(9): 092509.

\section{ISSUE DATE:}

2020-09

URL:

http://hdl.handle.net/2433/255549

\section{RIGHT:}

This article may be downloaded for personal use only. Any other use requires prior permission of the author and AIP Publishing. This article appeared in Physics of Plasmas 27, 092509 (2020) and may be found at https://doi.org/10.1063/5.0013652.; The full-text file will be made open to the public on 23 September 2021 in accordance with publisher's 'Terms and Conditions for Self-Archiving'. 


\section{Influence of plasma boundary shape on helical core/long-lived mode in tokamak plasmas}

Cite as: Phys. Plasmas 27, 092509 (2020); https://doi.org/10.1063/5.0013652

Submitted: 14 May 2020 . Accepted: 25 August 2020 . Published Online: 23 September 2020

Y. Nakamura, A. Ishizawa (D), and Y. Ishida (D)

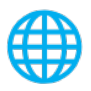

View Online

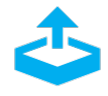

Export Citation

\section{ARTICLES YOU MAY BE INTERESTED IN}

Global gyrokinetic nonlinear simulations of kinetic infernal modes in reversed shear tokamaks Physics of Plasmas 27, 092302 (2020); https://doi.org/10.1063/5.0013349

Simulations of divertor plasmas with inverse sheaths

Physics of Plasmas 27, 092505 (2020); https://doi.org/10.1063/5.0015995

Kinetic simulations of anomalous resistivity in high-temperature current carrying plasmas

Physics of Plasmas 27, 092306 (2020); https://doi.org/10.1063/5.0004508

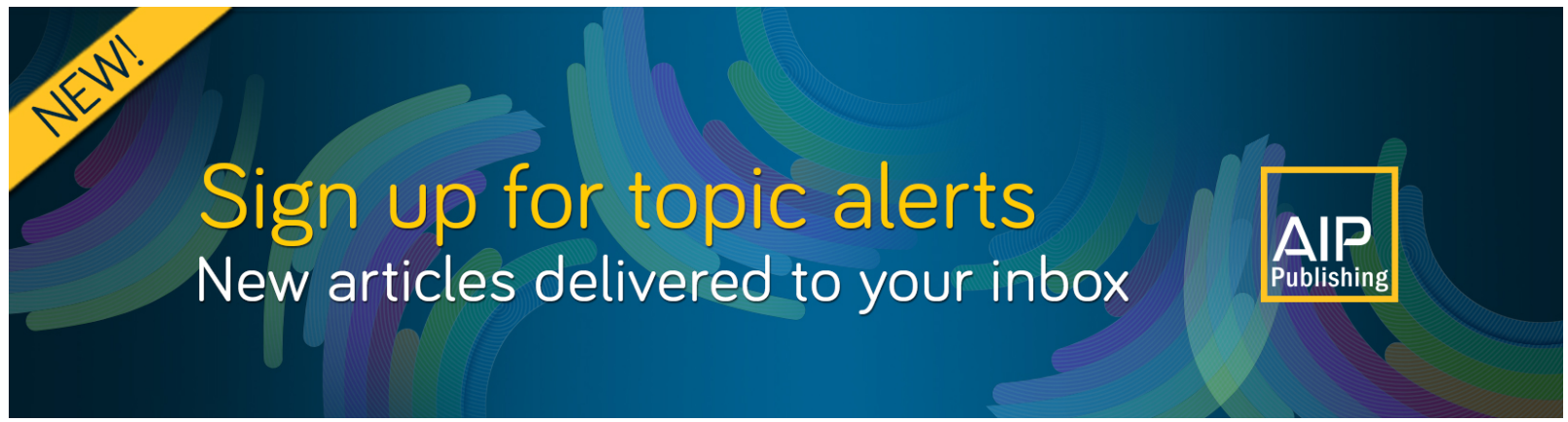




\title{
Influence of plasma boundary shape on helical core/long-lived mode in tokamak plasmas
}

\author{
Cite as: Phys. Plasmas 27, 092509 (2020); doi: 10.1063/5.0013652 \\ Submitted: 14 May 2020 - Accepted: 25 August 2020 . \\ Published Online: 23 September 2020
}

Y. Nakamura, ${ }^{\text {a) }}$ A. Ishizawa, (D) and Y. Ishida (D)

\author{
AFFILIATIONS \\ Graduate School of Energy Science, Kyoto University, Uji, Kyoto 611-0011, Japan
}

a) Author to whom correspondence should be addressed: nakamura@energy.kyoto-u.ac.jp

\begin{abstract}
Helical distortion of the core part of tokamak plasma, which is called a helical core or a long-lived mode, is investigated by means of three-dimensional magnetohydrodynamic equilibrium calculations. It is found that the magnitude of the helical distortion strongly depends on the shape of the plasma boundary for weakly reversed shear plasmas. The triangularity of the boundary enhances the amplitude of helical distortion. In addition, reversed $\mathrm{D}$-shape plasmas also exhibit a helical core. It is also found that the triangularity lowers the critical $\beta$ for the onset of a helical core; furthermore, the critical $\beta$ vanishes when the triangularity exceeds a certain value. On the other hand, the influence of the ellipticity on the amplitude of helical distortion strongly depends on $\beta$. The ellipticity enhances the amplitude at high $\beta$, while it reduces the amplitude at low $\beta$.
\end{abstract}

Published under license by AIP Publishing. https://doi.org/10.1063/5.0013652

\section{INTRODUCTION}

In tokamaks, good confinement is obtained by controlling the current density profile that is specified by the safety factor $q$ profile representing the twisting of magnetic field lines. For instance, the ITER hybrid scenario has a $q$-profile that is nearly flat and close to unity in the core region, ${ }^{1}$ leading to, for example, a better dependence of turbulent transport on $\beta$ because of weak magnetic shear in the core. $^{2}$ A helical distortion of the magnetic axis is observed for such a $q$-profile in some tokamaks, JET, MAST, and NCSX testing the hybrid scenario, ${ }^{3}$ that is called as the snake, ${ }^{4}$ the helical core, and the longlived mode. Analysis on helical cores was extended to DIII-D, Alcator C-Mod, and JT-60U. ${ }^{5-8}$ In the presence of a helical core, although the amplitude of helical displacement of plasma is largest at the magnetic axis, the displacement is finite even at the edge region, resulting in the fast-ion loss incorporated with toroidal ripple effects. ${ }^{3}$

The appearance of a helical core is understood as a bifurcation of a magnetohydrodynamic (MHD) equilibrium from an axisymmetric one to a non-axisymmetric one. ${ }^{9-13}$ The bifurcated equilibrium is regarded as a non-axisymmetric solution to the MHD equilibrium equation, which has an $(m, n)=(1,1)$ helical structure at the core, where $m$ and $n$ are the poloidal and toroidal mode numbers, respectively. It is remarked that this helical core structure appears, even when an axisymmetric boundary condition is imposed on the surface of the plasma. Numerical calculations of three-dimensional MHD equilibrium equation showed that helical structures appear when the safety factor $q$ profile has weakly reversed magnetic shear, the minimum of $q=q_{\min }$ is close to unity, and the volume averaged $\beta$ exceeds $0.7 \%$. Here, $\beta$ is the ratio of the plasma kinetic pressure to the magnetic pressure. In addition, a larger $q=q_{\text {min }}$ radius causes a larger helical displacement. ${ }^{6}$ The $(m, n)=(1,1)$ deformation mainly appears inside the $q=q_{\min } \approx 1$ radius, and thus it is related to internal $(m, n)$ $=(1,1)$ MHD modes ${ }^{14,15}$ and is called a long-lived mode. These MHD instabilities can be an internal kink mode and a quasi-interchange mode for a flat $q$-profile in the core region, ${ }^{14,15}$ and the correspondence between the helical core and the linear growth rate of $(m, n)=(1,1)$ mode is presented in Ref. 13. In addition, nonlinear saturated $(m, n)$ $=(1,1)$ instabilities are compared to the helical core. ${ }^{16}$

In this paper, we investigate the influence of plasma boundary shape on the helical distortion of plasmas to understand the conditions for helical core formation and the mechanism for the appearance of the bifurcated equilibrium. We mainly focus on the influence of the plasma boundary shape because it has a strong impact on the stability of the plasma, for instance, ellipticity (elongation) and triangularity of the plasma boundary are favorable for suppressing ballooning modes, ${ }^{17}$ which are an MHD instability driven by pressure gradient and appear in the unfavorable curvature region. On the other hand, a high ellipticity might lead to the vertical displacement event (VDE) during disruptions.

We solve the MHD equilibrium equation by using the VMEC code, ${ }^{19-21}$ which is a three-dimensional MHD equilibrium code, to 
evaluate the magnitude of the helical distortion of the magnetic axis and identify the conditions for the appearance of the bifurcated equilibrium. Motivated by the ITER hybrid scenario, ${ }^{22}$ which is expected to have weakly reversed shear and a large radius of the minimum of $q$, we consider a weakly reversed shear plasma that is same as that investigated in Ref. 9. We found that the conditions and the magnitude of helical distortion strongly depend on the plasma boundary shape. The magnitude increases with the triangularity, and the critical onset $\beta$ value decreases with the triangularity. We also found that a helical core appears even in the limit $\beta=0$ when the triangularity is large. On the other hand, the influence of the ellipticity strongly depends on $\beta$. The magnitude of helical distortion increases with the ellipticity at high $\beta$, while the magnitude decreases with the ellipticity at low $\beta$. The appearance of a helical core is limited to a range of $q_{\text {min }}$ around $q_{\text {min }} \approx 1$, and a larger amplitude of distortion tends to lead appearance over a wider range.

The organization of the remainder of this paper is as follows. Section II describes the model used in our numerical calculations. Section III presents the results of our calculations. We conclude with a summary of our results in Sec. IV.

\section{NUMERICAL MODEL AND SETTINGS}

We extend the analysis in Ref. 9 by introducing the influence of the plasma boundary shape. In this section, we describe the model used in our numerical calculations and our method for specifying the boundary shape of the plasma. We solve the MHD equilibrium equation by using the VMEC code which provides us an MHD equilibrium by minimizing the plasma potential energy

$$
W_{p}=\int\left(\frac{p}{\gamma-1}+\frac{B^{2}}{2 \mu_{0}}\right) d^{3} x
$$

by means of a steepest-decent method, where $\gamma$ is the specific heat ratio and is set to $\gamma=0$. We also impose a fixed boundary condition in this work. The influence of free boundaries is reported in Ref. 13, showing the influence is very minor. The cylindrical coordinates $(R, \zeta, Z)$ are generally represented by a Fourier series in terms of the flux coordinates $(s, \theta, \zeta)$ as

$$
\begin{aligned}
& R(s, \theta, \zeta)=\sum_{m} \sum_{n} R_{m n}(s) \cos (m \theta-n \zeta), \\
& Z(s, \theta, \zeta)=\sum_{m} \sum_{n} Z_{m n}(s) \sin (m \theta-n \zeta),
\end{aligned}
$$

where $s$ is the normalized toroidal flux. In this study, we assume that the boundary of plasma has up-down symmetry, and the plasma boundary shape $\left(R^{b}(\theta), Z^{b}(\theta)\right) \equiv(R(s=1, \theta, \zeta), Z(s=1, \theta, \zeta))$ is represented as

$$
\begin{gathered}
R^{b}(\theta)=R_{00}^{b}+R_{10}^{b} \cos \theta+R_{20}^{b} \cos 2 \theta, \\
Z^{b}(\theta)=Z_{10}^{b} \sin \theta .
\end{gathered}
$$

The Fourier coefficients are expressed in terms of the ellipticity $\kappa$, triangularity $\delta$, aspect ratio $A=R_{10}^{b} /\left(R_{00}^{b}+R_{20}^{b}\right)$, and $R_{00}^{b}$ as

$$
\begin{aligned}
& R_{10}^{b}=\frac{1}{A-\delta / 2} R_{00}^{b}, \\
& R_{20}^{b}=\frac{\delta / 2}{A-\delta / 2} R_{00}^{b},
\end{aligned}
$$

$$
Z_{10}^{b}=\frac{\kappa}{A-\delta / 2} R_{00}^{b}
$$

Our calculations are motivated by the ITER hybrid scenario, ${ }^{22}$ and we set $A=3$ and $R_{00}^{b}=5.6 \mathrm{~m}$ and focus on weakly reversed shear plasmas with a large $\rho_{\text {qmin }}$ in this work, where $\rho_{\text {qmin }}$ is the radius at $q=q_{\min }$. The pressure and $q$ profiles are given by

$$
p=p_{0}(1-s)
$$

and

$$
q=\left(0.7+0.7 s-s^{4}\right)^{-1},
$$

respectively, and they are the same profiles as those used in Ref. 9. We expect a large amplitude of the helical distortion for this $q$-profile which has the large radius of the minimum of safety factor $\rho_{\text {qmin }}$ because the amplitude of the helical distortion increases with increasing $\rho_{\text {qmin }}$ for weakly reversed shear as shown in Fig. 11 of Ref. 6 . The $q$-profile is shown in Fig. 1 and has a minimum value $q_{\min }=1.0064$ at $\rho_{\text {qmin }}=0.748$. The amplitude of the helical displacement of the magnetic axis is defined by the transverse displacement of the axis $R_{01}^{\mathrm{ax}} \equiv R_{01}(s=0, \theta, \zeta)$, and we use the normalized displacement

$$
\delta_{H} \equiv \frac{R_{01}^{\mathrm{ax}}}{a},
$$

where $a=R_{10}^{b}$ is the plasma minor radius.

\section{NUMERICAL RESULTS}

First, we show a typical helical core structure of a plasma. Figure 2 shows magnetic surfaces on cross sections at $\zeta=0, \pi / 4, \pi / 2$ and $3 \pi / 4$ for $\kappa=1.6$ and $\delta=0.172$, where $\zeta$ is the toroidal angle. As mentioned in the Introduction, the helical distortion has an $(m, n)$ $=(1,1)$ structure inside the $q\left(\rho_{\text {qmin }}\right)=q_{\min } \approx 1$ surface at $\rho_{\text {qmin }}$ $=0.748$ and can be related to an internal kink mode. ${ }^{16}$ Here, $\rho=\sqrt{s}$.

Next, we investigate the distortion of the magnetic axis quantitatively. In the following calculations, we use a sufficient number of radial grid points $N_{s}$ up to 3001 as discussed later. Figure 3(a) shows the distribution of the displacement $\delta_{H}$ with respect to the elongation and triangularity $\kappa-\delta$ plane for $\langle\beta\rangle=3 \%$. We have a helical distortion of the core on entire $\kappa-\delta$ plane, and the amplitude of the helical displacement $\delta_{H}$ increases with $\kappa$ and $\delta$, consistent with the $\kappa$ and $\delta$ dependence of the growth rate of internal kink modes. ${ }^{23,24}$ We also

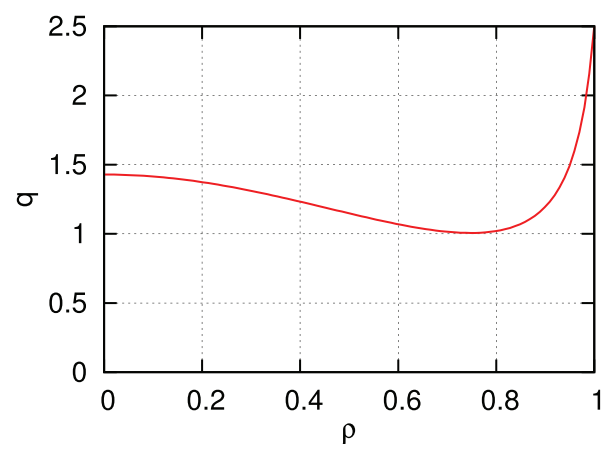

FIG. 1. Safety factor profile $q=\left(0.7+0.7 s-s^{4}\right)^{-1}$ as a function of $\rho=\sqrt{s}$, which has $q_{\min }=1.0064$ at $\rho_{\text {qmin }}=0.748$. 
Flux surfaces $\zeta=0 .[\mathrm{deg}]$

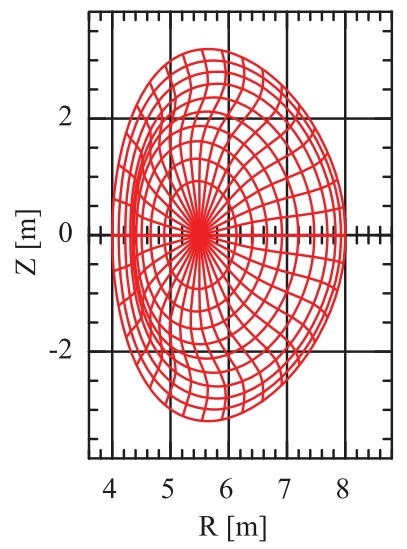

Flux surfaces $\zeta=90 .[\mathrm{deg}]$

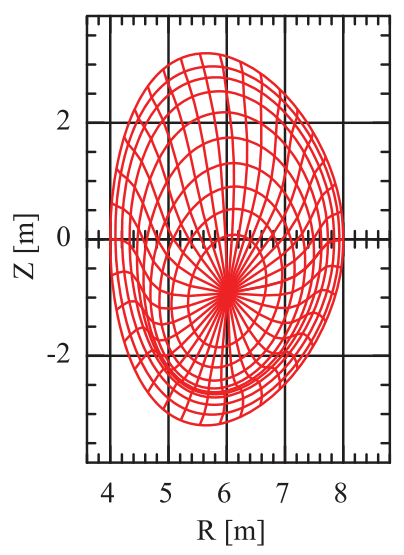

Flux surfaces $\zeta=45$. [deg]

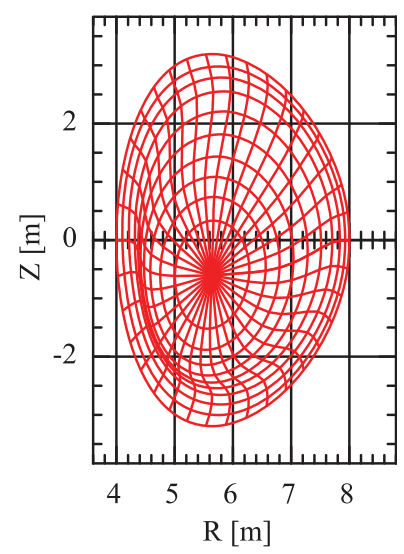

Flux surfaces $\zeta=135$.[deg]

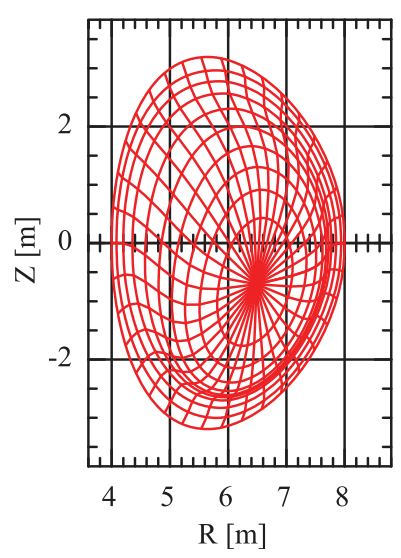

FIG. 2. Toroidal flux surfaces of a typical helical core/long-lived mode on cross sections at $\zeta=0, \pi / 4, \pi / 2$ and $3 \pi / 4$ for $\kappa=1.6$ and $\delta=0.172$.

have a helical distortion for reversed D-shape plasmas characterized by a negative triangularity $\delta<0$.

When we decrease $\beta$, asymmetry of the $\delta_{H}$ distribution with respect to $\delta$ and $\kappa$ on the $\delta-\kappa$ plane becomes prominent as shown in Figs. 3(b)-3(d). The amplitude of distortion becomes small at $\delta \simeq 0$ for $\langle\beta\rangle=2 \%$ in Fig. 3(b). The amplitude becomes further small at $\delta<0.1$ for $\langle\beta\rangle=1 \%$, while a large distortion remains at $\delta \geq 0.4$ in Fig. 3(c). For $\langle\beta\rangle=0$ limit, the distortion decreases with $\kappa$, while the large distortion still remains at $\delta \geq 0.4$ in Fig. 3(d).

The $\beta$ dependence of the amplitude of helical displacement of the magnetic axis $\delta_{H}$ is shown in Fig. 4, where $\langle\beta\rangle$ is the volume averaged $\beta$. The helical displacement increases with $\langle\beta\rangle$ regardless of the shape of the boundary, consistent with the $\beta$ dependence of the growth rate of internal kink modes. ${ }^{24}$ There is a critical $\langle\beta\rangle$ for a low triangularity $\delta=0$ and large ellipticity $\kappa=1.8$ and 2 as shown in Fig. 4(a), consistent with that shown in Ref. 9, while the $\beta$ dependence is very weak for small $\kappa$. When we increase triangularity, the critical $\langle\beta\rangle$ decreases, and there is no threshold for $\delta=0.4$ as shown in Fig. 4(b). The $\beta$ dependence of the helical displacement is weak for large $\delta$ as shown in Fig. 4(b), and the displacement almost does not depend on $\beta$ for small $\kappa$. Hence, there is no critical $\langle\beta\rangle$ for the formation of a helical core when the triangularity is large. It is remarked that increasing $\beta$ also causes the increase in pressure gradient $d p / d \rho$ in our calculations, and the importance of $d p / d \rho$ is reported in Ref. 6 .

The magnitude of the distortion strongly depends on the minimum value of the safety factor $q_{\text {min }}{ }^{3,9,10,13}$ In evaluating the dependence, here, $q_{\min }$ is varied by changing $\alpha$ in $q=(0.7+(0.7+\alpha) s$ $\left.-(1+\alpha) s^{4}\right)^{-1}$ with keeping $q(\rho=0)$ and $q(\rho=1)$ as is done in Ref. 9. The $q$-profile is shown in Fig. 5(a), and for instance, a minimum value is $q_{\text {min }}=1.055$ at $\rho_{\text {qmin }}=0.742$ for $\alpha=-0.1$ and $q_{\min }=$ 0.941 at $\rho_{\text {qmin }}=0.755$ for $\alpha=0.15$. Figures 5(b) -5 (d) show the amplitude of the helical displacement $\delta_{H}$ as a function of $q_{\min }$ for $\langle\beta\rangle=3 \%$ and $1 \%$. The helical core appears in $0.65<q_{\min }<1.07$, and the maximum amplitude of the helical distortion is in $q_{\text {min }} \approx 0.98$. For $\delta=0$, a higher $\kappa$ leads to a wider range of the appearance of the helical core and a higher amplitude of the helical displacement as shown in Fig. 5(b). When we increase the triangularity to $\delta=0.4$, as shown in Fig. 5(c), we have a wider range of the appearance of the helical core and a larger amplitude than that for $\delta=0$, while the range of the appearance is not so influenced by the ellipticity $\kappa$. On the other hand, when we decrease $\beta$ to $\langle\beta\rangle=1 \%$, we have a narrower range of the appearance of the helical core. The $\kappa$ dependence of the range of the appearance is weak and complicated. It is also remarked that we should be careful about the results for $q_{\min }<1$ in Fig. 5(b) because the appearance of $q=1$ rational surfaces might reduce the accuracy of the VMEC calculations due to the singularity of $(m, n)=(1,1)$ deformation at the rational surfaces.

Here, we present the difference of plasma potential energy between a bifurcated helical core solution and the corresponding axisymmetric solution to the MHD equilibrium equation. Figure 6 shows the normalized energy difference $\delta W_{H} \equiv\left(W_{\text {axi }}-W_{\text {hel }}\right) / W_{\text {axi }}$ on the $\kappa-\delta$ plane with $q=\left(0.7+0.7 s-s^{4}\right)^{-1}$, where $W_{\text {hel }}$ and $W_{\text {axi }}$ are the plasma potential energy of the helical core and axisymmetric solutions, respectively. The energy difference $\delta W_{H}$ for $\langle\beta\rangle=3 \%$ in Fig. 6(a) is positive, and its profile is similar to the profile of $\delta_{H}$ shown in Fig. 3(a); thus, a larger amplitude of helical core corresponds to a lower energy state. The energy difference has a minimum at $\kappa=\delta=0$, corresponding to the fact that the helical displacement has a minimum for a circular cross section as shown in Fig. 3(a). For the $\beta=0$ limit, the asymmetry of $\delta W_{H}$ distribution with respect to $\delta$ and $\kappa$ becomes prominent as shown in Fig. $6(\mathrm{~b})$, and $\delta W_{H}$ is very small at large $\kappa$ and small $\delta$. The distribution of $\delta W_{H}$ is similar to that of $\delta_{H}$ in Fig. 3(d). Thus, the helical core is a bifurcated solution which has a lower potential energy than the corresponding axisymmetric solution. It is expected that a helical core equilibrium having lower energy state is observed rather than the corresponding axisymmetric equilibrium in experiments.

Here, we examine the influence of aspect ratio $A$. Figure 7 shows the aspect ratio dependence of the helical distortion of the magnetic axis $\delta_{H}$ for $\langle\beta\rangle=3 \%$. The magnitude of the helical displacement decreases with increasing $A$. The $A$ dependence of $\delta_{H}$ is influenced by the value of $\kappa$ for $\delta=0$, while the $A$ dependence of $\delta_{H}$ is not so influenced by $\kappa$ for $\delta=0.4$. This is similar to the fact that the $\beta$ dependence of $\delta_{H}$ is strongly influenced by $\kappa$ for $\delta=0$, while it is not so influenced by $\kappa$ for $\delta=0.4$ in Fig. 4 . The influence of the aspect ratio $A$ on the $\beta$ dependence of the helical displacement $\delta_{H}$ is shown in Fig. 8. The helical displacement decreases with increasing the aspect 
(a) Helical displacement $\delta_{\mathrm{H}}<\beta>=3 \%$

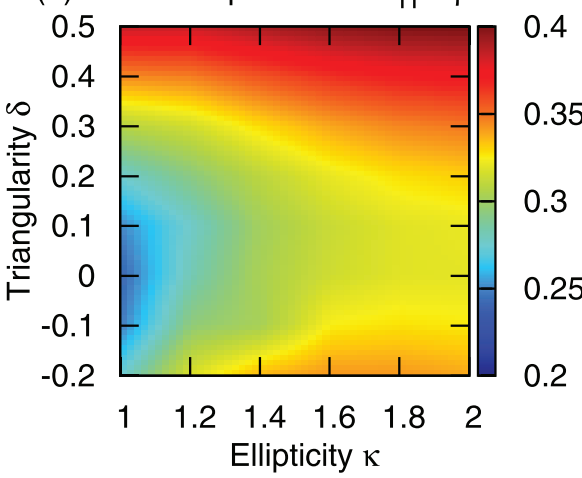

(c) Helical displacement $\delta_{H}<\beta>=1 \%$

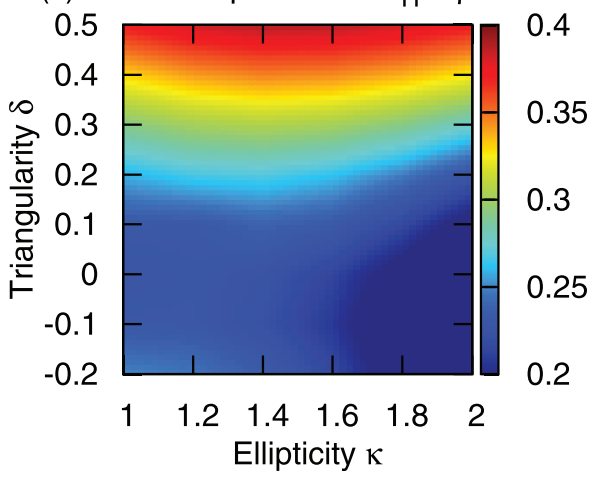

(b) Helical displacement $\delta_{\mathrm{H}}<\beta>=2 \%$

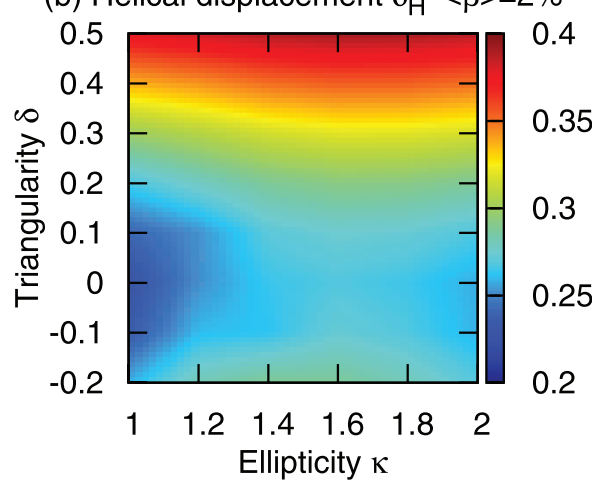

(d) Helical displacement $\delta_{H}<\beta>=0 \%$

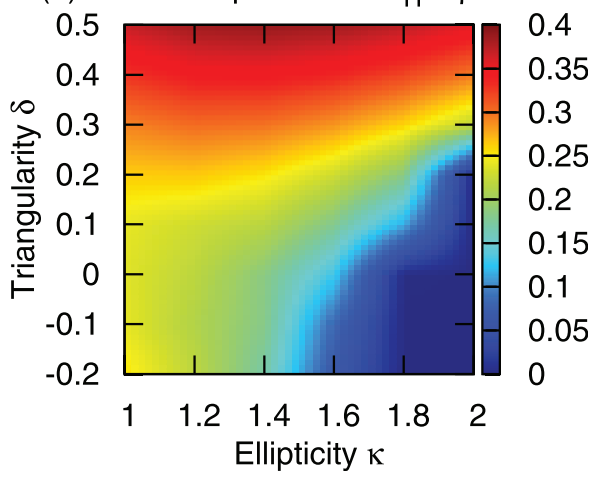

FIG. 3. The amplitude of helical displacement of the magnetic axis $\delta_{H}$ on the elongation and triangularity $\kappa-\delta$ plane for (a) $\langle\beta\rangle=3 \%$, (b) $\langle\beta\rangle=2 \%$, (c) $\langle\beta\rangle=1 \%$, and (d) $\langle\beta\rangle=0 \%$ with $q=\left(0.7+0.7 s-s^{4}\right)^{-1}$ that has $q_{\min }=1.0064$ at $\rho_{\text {qmin }}=0.748$.

ratio for all cases in Fig. 8 except for reversed D-shape plasmas. The $\beta$ dependence of $\delta_{H}$ is weak for small $\delta$ and small $\kappa$ as shown in Fig. 8(a), consistent with the results in Fig. 4(a). The $\beta$ dependence is strong for small $\delta$ and large $\kappa$ as shown in Fig. 8(b). We have a critical onset $\beta$ value for the formation of a helical core for $A=3$ and 3.5 , and the critical $\beta$ increases with $A$. For large $\delta$ and large $\kappa$, the $\beta$ dependence of $\delta_{H}$ is weak, and there is no critical onset $\beta$ as shown in Fig. 8 (c). For reversed D-shape plasmas, the $\beta$ dependence of $\delta_{H}$ is strong,
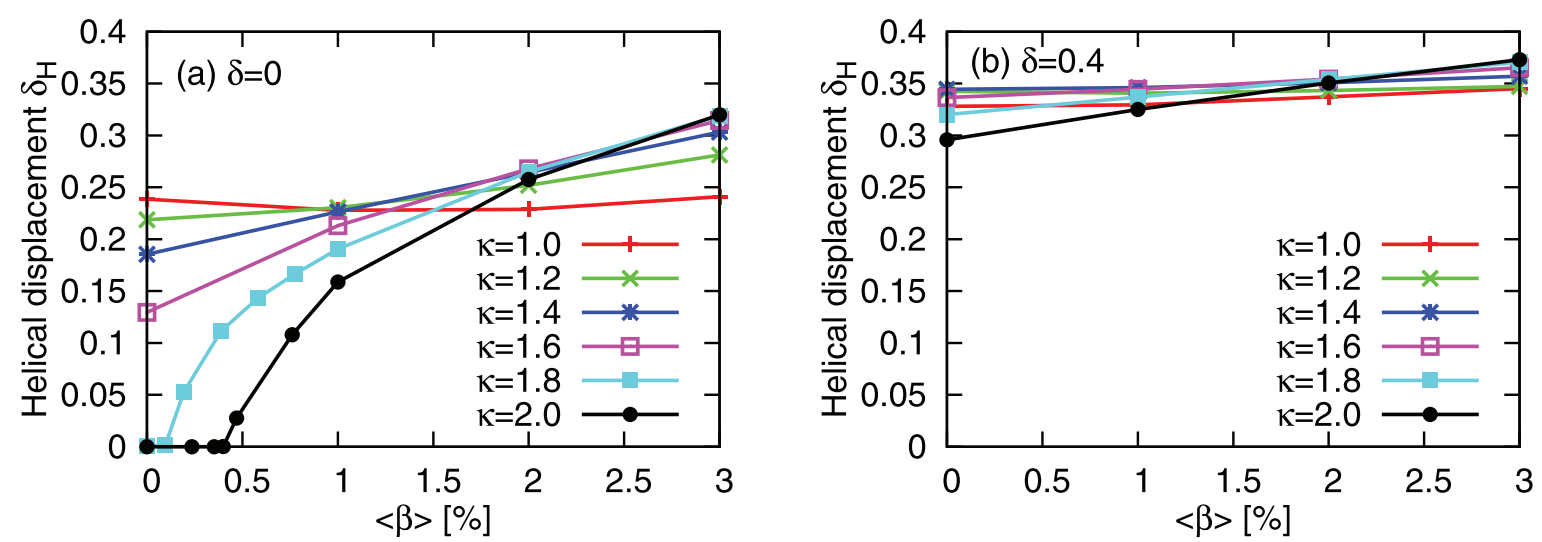

FIG. 4. The amplitude of helical displacement of the magnetic axis $\delta_{H}$ as a function of the volume averaged beta $\langle\beta\rangle$ for the triangularity (a) $\delta=0$ and (b) $\delta=0.4$ with $q=$ $\left(0.7+0.7 s-s^{4}\right)^{-1}$ that has $q_{\min }=1.0064$ at $\rho_{\text {qmin }}=0.748$. 

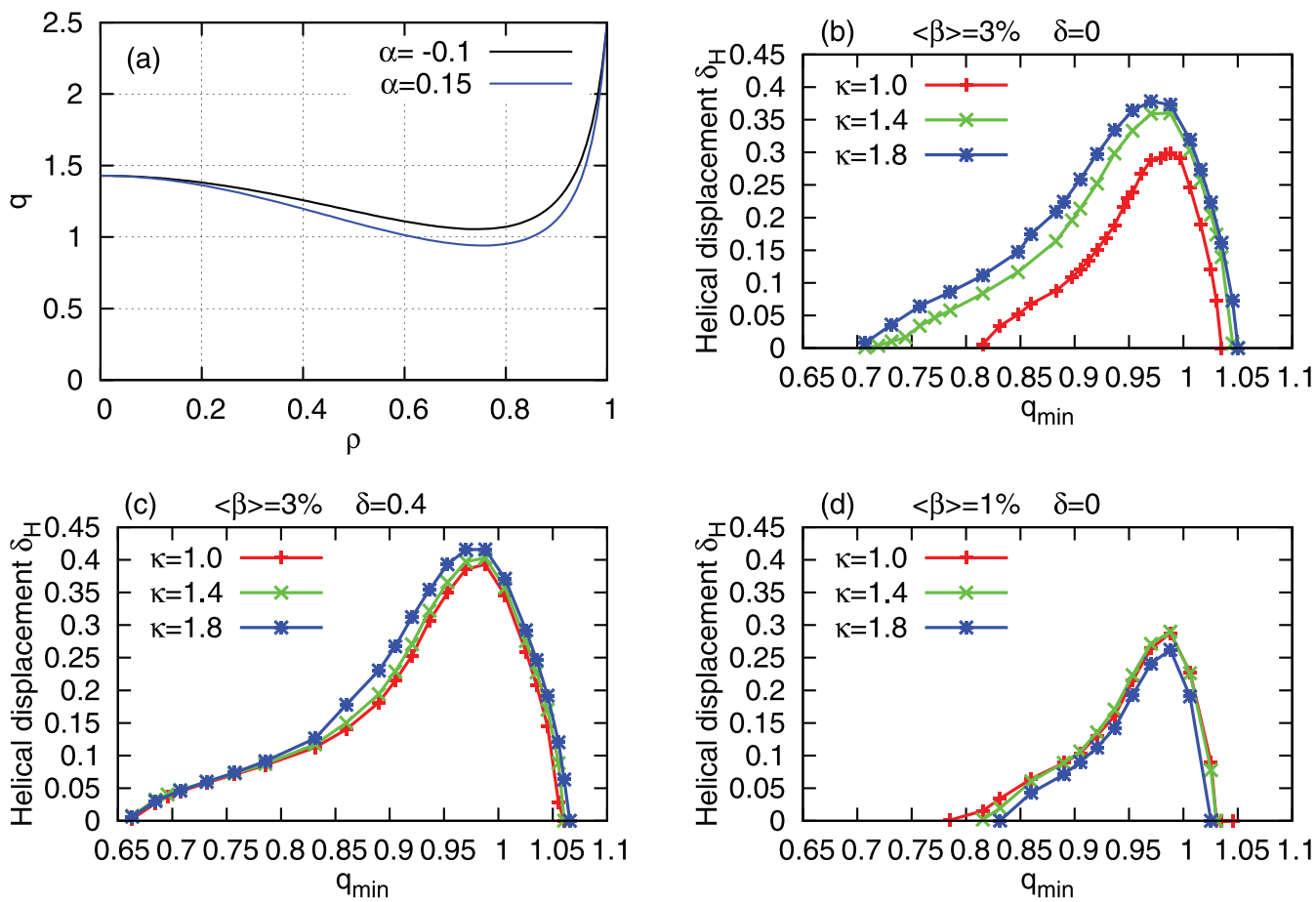

FIG. 5. (a) Safety factor profile $q=\left[0.7+(0.7+\alpha) s-(1+\alpha) s^{4}\right]^{-1}$ used in evaluating $q_{\min }$ dependence. The amplitude of helical displacement of the magnetic axis $\delta_{H}$ as a function of the minimum value of safety factor $q_{\min }$ for (b) $\langle\beta\rangle=3 \%$ and $\delta=0$, (c) $\langle\beta\rangle=3 \%$ and $\delta=0.4$, and (d) $\langle\beta\rangle=1 \%$ and $\delta=0$.

whereas the aspect ratio dependence of $\delta_{H}$ is weak as shown in Fig. 8 (d). We have a critical onset $\beta$ value for the formation of a helical core for $A=2.5,3$, and 3.5 , and the critical $\beta$ slightly increases with $A$.

Finally, we discuss the dependence of the helical displacement on the number of radial grid points. Figure 9 shows the amplitude of helical displacement of the magnetic axis $\delta_{H}$ as a function of the number of radial grid points $N_{s}$. For $(\delta, \kappa)=(0.4,1.8)$ and $\langle\beta\rangle=3 \%$, the helical displacement is large $\delta_{H}=0.37$, and $N_{s}=200$ is large enough to obtain a reliable $\delta_{H}$, whereas, for $(\delta, \kappa)=(-0.2,1.6)$ and $\langle\beta\rangle=0$, the helical displacement is small $\delta_{H}=0.074$, and $N_{s}=3000$ is needed to obtain a reliable $\delta_{H}$. Similar $N_{s}$ dependence of $\delta_{H}$ is shown and $N_{s}=193$ is employed for the analysis of helical ITER equilibrium in Ref. 10 , and $N_{s}=3000$ is used for some calculations in Ref. 6. Thus, a large $N_{s}$ is required to obtain an accurate amplitude of helical displacement when the amplitude $\delta_{H}$ is small. This dependence on the number of radial grid points is responsible for the analysis of $q_{\min }$ dependence of $\delta_{H}$ even when we have large helical displacement at $q_{\min } \approx 1$. Figure 10 shows the amplitude of helical displacement of the magnetic
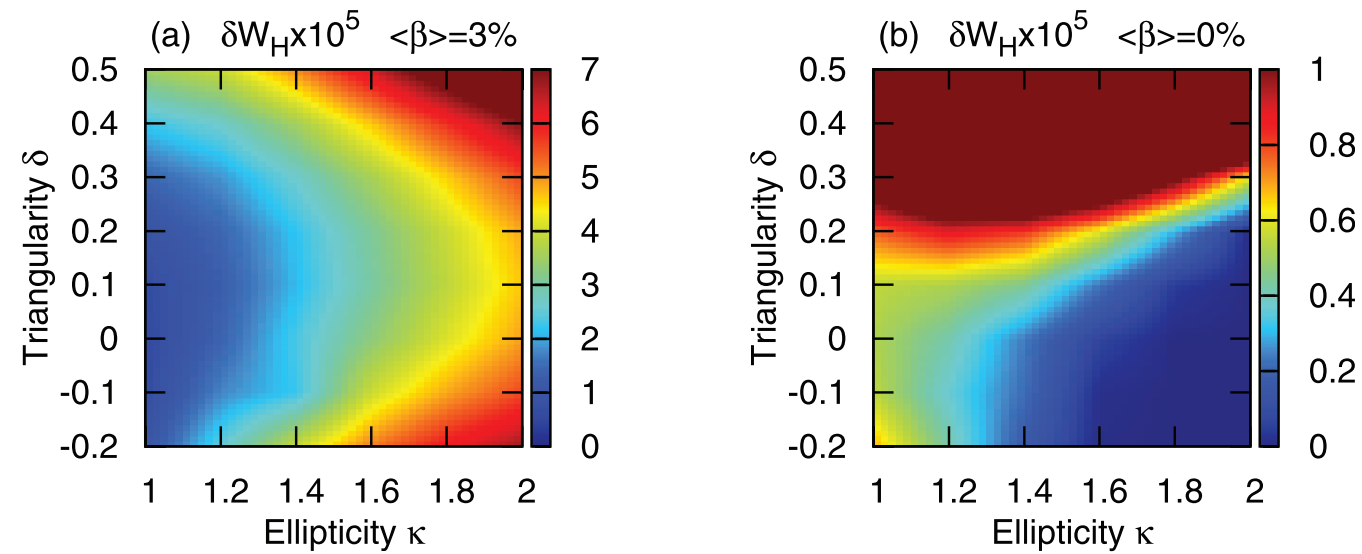

FIG. 6. The normalized difference of plasma potential energy between helical core and axisymmetric equilibria $\delta W_{H} \equiv\left(W_{\text {axi }}-W_{\text {hel }}\right) / W_{\text {axi }}$ on the $\kappa-\delta$ plane for the volume averaged $\beta$ (a) $\langle\beta\rangle=3 \%$ and (b) $\langle\beta\rangle=0 \%$ with $q=\left(0.7+0.7 s-s^{4}\right)^{-1}$ that has $q_{\min }=1.0064$ at $\rho_{\text {qmin }}=0.748$. 

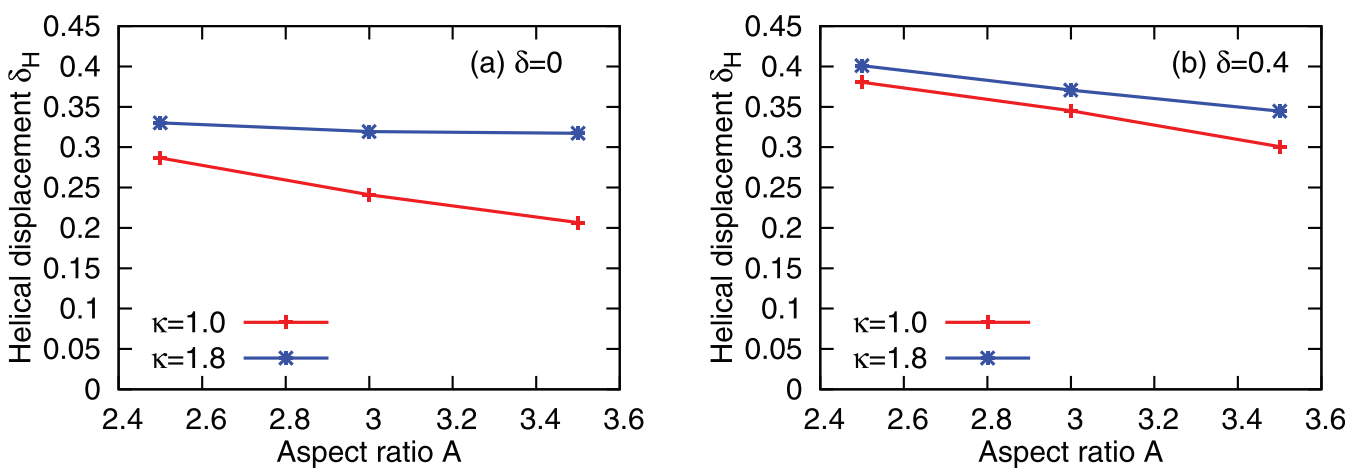

FIG. 7. The amplitude of helical displacement of the magnetic axis $\delta_{H}$ as a function of aspect ratio $A$ for (a) $\delta=0$ and (b) $\delta=0.4$ with $\langle\beta\rangle=3 \%$ and $q=$ $\left(0.7+0.7 s-s^{4}\right)^{-1}$ that has $q_{\min }=1.0064$ at $\rho_{\text {qmin }}=0.748$.

axis $\delta_{H}$ as a function of $q_{\min }$ for several numbers of radial grid points $N_{s}$, which corresponds to $\delta_{H}$ in Fig. 5 (c) with $\langle\beta\rangle=3 \%, \delta=0.4$, and $\kappa=1.8$. When $q_{\min }>1$, we obtain a reliable $\delta_{H}$ for $N_{s}>200$ as shown in Fig. 9. On the other hand, a large number of grid points is needed for $q_{\min }<1$, and $N_{s}=3001$ is required to obtain an accurate $\delta_{H}$ for $q_{\min }<0.8$, because a large number of grid points are necessary for obtaining an accurate helical displacement for small displacement as shown in Fig. 9. When we compare $\delta_{H}$ with the linear growth rate of internal $(m, n)=(1,1)$ MHD instability of the corresponding axisymmetric equilibrium, a large number of grid points is necessary for the analysis of plasmas with $q_{\text {min }}<1$. It is expected that the $q_{\text {min }}$ dependence of $\delta_{H}$ obtained by using a sufficiently large $N_{s}$ at $q_{\min }<1$ is close to the corresponding $q_{\text {min }}$ dependence of the linear growth rate because a large $N_{s}$ allows us to capture the rapid change of an $(m, n)=(1,1)$ plasma displacement at $q=1$ rational surface.

\section{SUMMARY AND DISCUSSION}

We have investigated the influence of plasma boundary shape on the conditions for onset of a helical core and on the amplitude of the helical displacement of the magnetic axis for weakly reversed shear
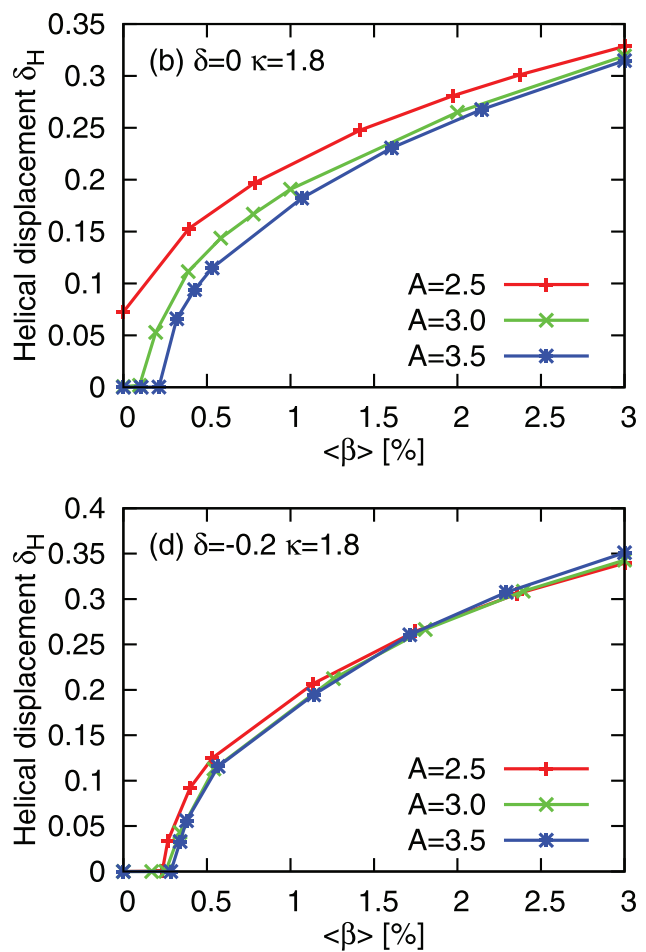

FIG. 8. The amplitude of helical displacement of the magnetic axis $\delta_{H}$ as a function of $\langle\beta\rangle$ for several aspect ratio $A$ : (a) $(\delta, \kappa)=(0,1.0)$, (b) $(\delta, \kappa)=(0,1.8)$, (c) $(\delta, \kappa)=(0.4,1.8)$, and $(\mathrm{d})(\delta, \kappa)=(-0.2,1.8)$ with $q=\left(0.7+0.7 s-s^{4}\right)^{-1}$ that has $q_{\min }=1.0064$ at $\rho_{\text {qmin }}=0.748$. 


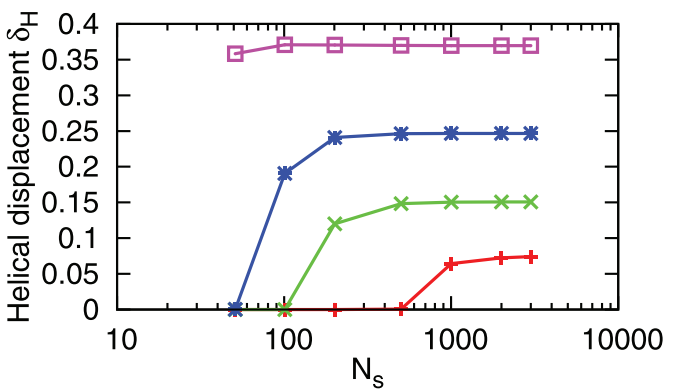

\begin{tabular}{llll|}
$\delta=-0.2$ & $\kappa=1.6$ & $<\beta>=0[\%]$ \\
$\delta=-0.2$ & $\kappa=2.0$ & $<\beta>=1[\%]$ \\
$\delta=0.0$ & $\kappa=1.0$ & $<\beta>=3[\%]$ \\
$\delta=0.4$ & $\kappa=1.8 \quad<\beta>=3[\%]$ \\
\hline
\end{tabular}

FIG. 9. The amplitude of helical displacement of the magnetic axis $\delta_{H}$ as a function of radial grid number $N_{s}$ for $A=3$ with $q=\left(0.7+0.7 s-s^{4}\right)^{-1}$ that has $q_{\min }$ $=1.0064$ at $\rho_{\text {qmin }}=0.748$.

plasmas. We evaluated the magnitude of helical distortion by means of MHD equilibrium calculations and examined its dependence on the plasma boundary shape in terms of the ellipticity $\kappa$ and triangularity $\delta$. We also examined the dependence on the volume averaged beta $\langle\beta\rangle$ and the minimum value of the safety factor $q_{\text {min }}$.

The amplitude of helical distortion strongly depends on the boundary shape. The amplitude increases with increasing the triangularity $\delta$. In addition, the reversed D-shape $(\delta<0)$ also exhibits the helical core. On the other hand, the influence of the ellipticity $\kappa$ strongly depends on the $\beta$ value. The amplitude increases with increasing the ellipticity $\kappa$ at high $\beta$, whereas the amplitude decreases with increasing $\kappa$ at low $\beta$. The larger the helical distortion, the lower the plasma potential energy, indicating that the helical core is a bifurcated equilibrium that has a lower plasma potential energy. The $\beta$ value is one of the important parameters controlling the amplitude of helical core, ${ }^{9}$ and we have found that the $\beta$ dependence of the amplitude is also strongly influenced by the plasma boundary shape. For small triangularity, the amplitude increases with increasing $\beta$, and the

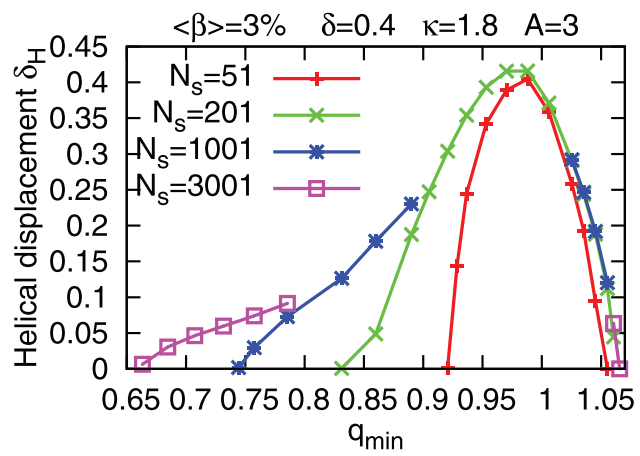

FIG. 10. The amplitude of helical displacement of the magnetic axis $\delta_{H}$ as a function of the minimum value of safety factor $q_{\min }$ for several values of radial grid number $N_{s}$ with $\langle\beta\rangle=3 \%, \delta=0.4, \kappa=1.8, A=3$, and $q=[0.7+(0.7+\alpha) s$ $\left.-(1+\alpha) s^{4}\right]^{-1}$. amplitude becomes very small at low $\beta$, and thus we have a critical $\beta$ for the formation of a helical core when the triangularity is sufficiently small. For large triangularity, by contrast, the threshold disappears and the $\beta$ dependence is very weak, so that the helical core appears even for $\beta=0$ limit. The above results are obtained for $q_{\min }>1$.

The helical core formation also depends on $q_{\text {min }}$, and the helical core appears at $q_{\min } \approx 1$. The maximum of the distortion is located at $q_{\text {min }}<1$, consistent with that shown in Refs. 3, 9, 10, and 13. As the mechanism of the appearance of a helical core, $(m, n)=(1,1)$ MHD instabilities such as internal kink modes and quasi-interchange modes in a flat $q$-profile ${ }^{14,15}$ are suggested by the fact that the amplitude $\delta_{H}$ has a maximum around $q_{\min }=1$ regardless of the boundary shape. In addition, a larger triangularity leads to a larger helical distortion, consistent with a higher linear growth rate of internal kink modes for a larger triangularity in a weakly reversed shear plasma in Fig. 13 of Ref. 24. The linear growth rate can be related to the saturated amplitude of $(m, n)=(1,1)$ mode. $^{25}$ In order to clarify the relation between helical cores and the MHD instabilities, we will calculate the linear stability of axisymmetric equilibria corresponding to the helical core equilibria and will report the correspondence between the linear growth rate and the amplitude of helical displacement in our next paper.

\section{ACKNOWLEDGMENTS}

This work was supported by the Japanese Ministry of Education, Culture, Sports, Science and Technology, Grant No. $18 \mathrm{~K} 03583$.

\section{DATA AVAILABILITY}

The data that support the findings of this study are available from the corresponding author upon reasonable request.

\section{REFERENCES}

${ }^{1}$ E. Joffrin, F. Crisanti, R. Felton, X. Litaudon, D. Mazon, D. Moreau, L. Zabeo, R. Albanese, M. Ariola, D. Alves, O. Barana, V. Basiuk et.al., Plasma Phys. Controlled Fusion 45, A367 (2003).

${ }^{2}$ A. Ishizawa, D. Urano, Y. Nakamura, S. Maeyama, and T.-H. Watanabe, Phys. Rev. Lett. 123, 025003 (2019).

${ }^{3}$ I. T. Chapman, D. Brunetti, P. Buratti, W. A. Cooper, J. P. Graves, J. R. Harrison, J. Holgate, S. Jardin, S. A. Sabbagh, K. Tritz, and MAST and NSTX Teams and EFDA-JET Contributors, Nucl. Fusion 54, 083007 (2014).

${ }^{4}$ A. Weller, A. D. Cheetham, A. W. Edwards, R. D. Gill, A. Gondhalekar, R. S. Granetz, J. Snipes, and J. A. Wesson, Phys. Rev. Lett. 59, 2303 (1987).

${ }^{5}$ M. Cianciosa, A. Wingen, S. P. Hirshman, S. K. Seal, E. A. Unterberg, R. S. Wilcox, P. Piovesan, L. Lao, and F. Turco, Nucl. Fusion 57, 076015 (2017).

${ }^{6}$ A. Wingen, R. S. Wilcox, S. K. Seal, E. A. Unterberg, M. R. Cianciosa, L. F. Delgado-Aparicio, S. P. Hirshman, and L. L. Lao, Nucl. Fusion 58, 036004 (2018).

${ }^{7}$ A. Wingen, R. S. Wilcox, L. F. Delgado-Aparicio, R. Granetz, S. Houshmandyar, S. Shiraiwa, M. R. Cianciosa, and S. K. Seal, Phys. Plasmas 26, 022501 (2019)

${ }^{8}$ T. Bando, G. Matsunaga, M. Takechi, A. Isayama, N. Oyama, S. Inoue, M. Yoshida, and T. Wakatsuki, Plasma Phys. Controlled Fusion 61, 115014 (2019).

${ }^{9}$ W. A. Cooper, J. P. Graves, A. Pochelon, O. Sauter, and L. Villard, Phys. Rev. Lett. 105, 035003 (2010).

${ }^{10}$ W. A. Cooper, J. P. Graves, and O. Sauter, Plasma Phys. Controlled Fusion 53, 024002 (2011).

${ }^{11}$ W. A. Cooper, J. P. Graves, O. Sauter, I. T. Chapman, M. Gobbin, L. Marrelli, P. Martin, I. Predebon, and D. Terranova, Plasma Phys. Controlled Fusion 53, 074008 (2011). 
${ }^{12}$ W. A. Cooper, J. P. Graves, and O. Sauter, Nucl. Fusion 51, 072002 (2011).

${ }^{13}$ W. A. Cooper, J. P. Graves, O. Sauter, J. Rossel, M. Albergante, S. Coda, B. P. Duval, B. Labit, A. Pochelon, H. Reimerdes, and TCV Team, Plasma Phys. Controlled Fusion 53, 124005 (2011).

${ }^{14}$ M. N. Bussac, R. Pellat, D. Edery, and J. L. Soule, Phys. Rev. Lett. 35, 1638 (1975).

${ }^{15}$ J. A. Wesson, Plasma Phys. Controlled Fusion 28, 243 (1986).

${ }^{16}$ D. Brunetti, J. P. Graves, W. A. Cooper, and D. Terranova, Nucl. Fusion 54, 064017 (2014)

${ }^{17} \mathrm{~J}$. Freidberg, Ideal MHD (Cambridge University Press, 2014).

${ }^{18}$ O. Gruber, K. Lackner, G. Pautasso, U. Seidel, and B. Streibl, Plasma Phys. Controlled Fusion 35, B191 (1993).

${ }^{19}$ S. P. Hirshman and J. C. Whitson, Phys. Fluids 26, 3553 (1983).
${ }^{20}$ S. P. Hirshman, W. I. van Rij, and P. Merkel, Comput. Phys. Commun. 43, 143 (1986).

${ }^{27}$ S. P. Hirshman and H. K. Meier, Phys. Fluids 28, 1387 (1985).

${ }^{22}$ E. Strumberger, S. Gunter, P. Merkel, E. Schwarz, and C. Tichmann, Nucl. Fusion 50, 025008 (2010).

${ }^{23}$ J. P. Graves, C. Angioni, R. V. Budny, R. J. Buttery, S. Coda, L.-G. Eriksson, C. G. Gimblett, T. P. Goodman, R. J. Hastie, M. A. Henderson, H. R. Koslowski, M. J. Mantsinen, A. Martynov, M.-L. Mayoral, A. Muck, M. F. F. Nave, O. Sauter, E. Westerhof, and JET-EFDA Contributors, Plasma Phys. Controlled Fusion 47, B121 (2005).

${ }^{24}$ R. J. Hastie and T. C. Hender, Phys. Fluids 30, 1756 (1987).

${ }^{25}$ J. P. Graves, D. Zullino, D. Brunetti, S. Lanthaler, and C. Wahlberg, Plasma Phys. Controlled Fusion 61, 104003 (2019). 